\title{
Ressources en ligne. L'intégration scolaire des enfants de migrants et du voyage
}

Sophie Condat

\section{(2) OpenEdition \\ 1 Journals}

Édition électronique

URL : http://journals.openedition.org/ries/1180

DOI : 10.4000/ries. 1180

ISSN : 2261-4265

Éditeur

Centre international d'études pédagogiques

Édition imprimée

Date de publication : 1 septembre 2005

Pagination : 10-12

ISBN : 978-2-85420-564-0

ISSN : $1254-4590$

Référence électronique

Sophie Condat, «Ressources en ligne. L'intégration scolaire des enfants de migrants et du voyage », Revue internationale d'éducation de Sèvres [En ligne], 39 | septembre 2005, mis en ligne le 17 novembre 2011, consulté le 30 avril 2019. URL : http://journals.openedition.org/ries/1180 ; DOI : 10.4000/ ries. 1180

Ce document a été généré automatiquement le 30 avril 2019

(c) Tous droits réservés 


\title{
Ressources en ligne. L'intégration scolaire des enfants de migrants et du voyage
}

\author{
Sophie Condat
}

1 La sélection de sites Internet proposée ici, non exhaustive, arrêtée en juin 2005, mentionne les dispositifs officiels existants pour faciliter l'intégration scolaire des enfants de migrants et des enfants du voyage. Dans la plupart des pays européens, les jeunes étrangers représentent environ $6 \%$ de la population en âge scolaire (Eurostat). Nous avons choisi de nous intéresser à l'Europe où l'intégration des immigrés fait partie des débats politiques actuels, à travers les recommandations du Conseil de l'Europe et les politiques menées en Europe; au cas particulier de la France; à la situation d'un pays d'Amérique, le Canada, terre d'immigration importante.

\section{L'accueil et la scolarisation des enfants de migrants}

\section{Les politiques d'intégration scolaire en Europe}

Depuis sa création, le Conseil de l'Europe porte une attention particulière aux politiques d'intégration des immigrés. Il recommande d'adapter le système éducatif aux besoins des enfants immigrants, d'intégrer des cours de langue et de culture du pays d'origine et de promouvoir l'éducation interculturelle pour tous. Dans un document sur l'accueil des immigrants, le rapport de l'Assemblée parlementaire énumère les politiques d'intégration des immigrés dans les États membres du Conseil de l'Europe (2003), http:// assembly.coe.int

\section{L'enquête d'Eurydice}

3 Eurydice, le réseau d'information sur l'éducation en Europe, a analysé en 2004 les mesures scolaires mises en place dans les pays d'accueil pour les enfants de migrants. Les données concernent les niveaux pré-primaire, primaire et secondaire général obligatoire. 
Cette analyse, présentée aussi sous la forme d'un cédérom, compare la situation de trente pays d'Europe à travers six thématiques: la politique européenne en ce domaine, la situation démographique, les droits à l'éducation et aux dispositifs de soutien, les dispositifs d'intégration scolaire, la prise en compte de la culture d'origine des élèves immigrants et l'approche interculturelle à l'école. Un glossaire, des annexes (des documents de référence sur les politiques et la législation de l'Union européenne, des textes du Conseil de l'Europe) ainsi qu'une sélection de références bibliographiques et de sites Internet complètent l'étude. http://www.eurydice.org

4 L'enquête est également accessible par pays et précise les définitions nationales, le contexte démographique, les mesures de soutien scolaire, la prise en compte de l'approche culturelle dans l'éducation, l'évaluation, les projets pilotes, les débats et réformes prévues. http://www.eurydice.org

\section{Accueil et scolarisation des élèves nouvellement arrivés en France}

539100 nouveaux arrivants non francophones ont été accueillis en France durant l'année scolaire 2003-2004. La problématique de l'immigration a évolué et, avec elle, les structures d'accueil, leurs missions et leurs dénominations. La Cour des comptes a publié en 2004 un rapport sur L'accueil des immigrants et l'intégration des populations issues de l'immigration. Une partie concerne l'école. http://lesrapports.ladocumentationfrancaise.fr

\section{Les dispositifs d'accueil et de scolarisation}

Le Haut conseil à l'intégration a remis en 2003 un rapport au Premier ministre intitulé Le contrat et l'intégration. Il insiste sur l'attention particulière qu'il convient d'apporter aux jeunes immigrés ainsi que sur les solutions spécifiques que leur intégration nécessite. http://www.ladocumentationfrancaise.fr

7 Le CNDP propose sur son site une rubrique spécifique, "savoirscdi ", qui présente un dossier sur la scolarisation des enfants nouvellement arrivés en France. Il fait le point sur les dispositifs existants ainsi que sur les textes en vigueur et les outils disponibles: http://savoirscdi.cndp.fr/pedago

8 Une circulaire du ministère de l'Éducation nationale de 2002 définit les missions et l'organisation des centres académiques pour la scolarisation des nouveaux arrivants et des enfants du voyage (CASNAV): http://www.education.gouv.fr/index.php . Les publications du CASNAV sont téléchargeables au format pdf: Accompagner un élève non francophone: Logiciels éducatifs (2004), Accueillir et scolariser les enfants tsiganes et voyageurs en classe ordinaire : document d'accueil (2002), etc. http://www.ac-nancy-metz.fr/cefisem

9 Le centre de ressources Ville-École-Intégration (VEI) du SCÉRÉN - CNDP propose un dossier sur l'accueil et la scolarisation des élèves nouvellement arrivés en France : un glossaire (des définitions relatives au français langue seconde), le descriptif des systèmes éducatifs étrangers, des évaluations, des outils et des méthodes pédagogiques, des expériences innovantes et des conseils de lecture. http://www.cndp.fr/vei/acc_scol/ accueil.htm 


\section{Au Canada}

10 En 2001, plus du quart des jeunes de 5 à 24 ans vivant à Toronto et à Vancouver avaient immigré au Canada dans les années 1990. Une étude de Statistique Canada (mise à jour en 2004) s'intitule Les Enfants d'immigrants: comment se débrouillent-ils à l'école? http:// www.statcan.ca

11 Au Québec, une série de mesures destinées aux élèves allophones vise à faciliter leur intégration. http://www.infobourg.com

«Immigration et métropoles", site du Centre de recherche interuniversitaire de Montréal sur l'immigration, l'intégration et la dynamique urbaine, a développé un projet sur la réussite scolaire et la mobilité éducative des élèves d'origine immigrée, http:// im.metropolis.net/frameset_f.html

\section{La scolarisation des enfants du voyage}

13 La problématique de l'intégration scolaire des enfants roms / tsiganes diffère de celle des enfants de migrants, notamment en raison du taux élevé d'analphabétisme ou de semianalphabétisme de cette communauté, de l'ampleur de l'échec scolaire, de la faible proportion de jeunes achevant leurs études primaires et de la persistance de facteurs tels que l'absentéisme scolaire.

\section{Le projet du Conseil de l'Europe}

Le projet Éducation des enfants roms/tsiganes en Europe met en œuvre la Recommandation $n^{\circ} R(2000) 4$ du Comité des ministres aux États membres sur l'éducation des enfants roms/tsiganes en Europe. Elle stipule que l'éducation de ces enfants doit être une priorité des politiques nationales menées en faveur de ces populations. http:// www.hri.ca/

15 Le Conseil de l'Europe propose par ailleurs sur son site un répertoire des textes officiels, des activités (séminaires de réflexion, stages de formation, production de matériel pédagogique, etc.) et également des publications. http://www.coe.int/

\section{La scolarisation des enfants du voyage en France}

16 Au cours des dernières années, on a constaté une hausse de la fréquentation scolaire des enfants de familles non sédentaires, tout particulièrement au niveau de l'école primaire.

Le ministère de l'Éducation nationale a édicté en 2002 une circulaire sur la scolarisation des enfants du voyage et de familles non sédentaires. Elle donne des recommandations pour l'organisation et le suivi de leur scolarisation. http://www.education.gouv.fr

VEI propose un dossier sur la scolarisation des enfants du voyage et des familles non sédentaires : textes, informations sur les populations tsiganes ; description des dispositifs de scolarisation, des outils et des méthodes d'enseignement; expériences; projets français et européens ; références bibliographiques ; sources d'informations spécialisées. http://www.cndp.fr/vei/enfvoyage/accueil.htm 
19 L'Institut national d'études démographiques (INED) et la direction de l'animation, de la recherche et des études statistiques (DARES) ont organisé en 2005 un colloque sur l'insertion scolaire et professionnelle des jeunes issus de l'immigration. Les textes des intervenants sont téléchargeables sur http://dares.ptolemee.com/Synthese18mars.pdf

\section{AUTEUR}

\section{SOPHIE CONDAT}

Documentaliste, centre de ressources du CIEP 